\title{
Pacific
}

Journal of

Mathematics

\section{REFLEXIVITY OF SUBNORMAL OPERATORS}

JOHN MCCARTHY

Volume $161 \quad$ No. 2

December 1993 


\title{
REFLEXIVITY OF SUBNORMAL OPERATORS
}

\author{
JOHN E. MCCARTHY
}

Dedicated to Donald Sarason, in admiration of the range of his pioneering work

We give a new proof that subnormal operators are reflexive. We extend this to certain subnormal $n$-tuples. We give the first complete proof that a pair of doubly commuting isometries is reflexive.

0. Introduction. Let $A$ be a weakly closed algebra of bounded linear operators on a Hilbert space $\mathscr{H}$. Its lattice, $\operatorname{Lat}(A)$, is the set of all closed subspaces of $\mathscr{H}$ that are left invariant by every element of $A$. The set of operators that leave invariant every space in Lat $(A)$ is denoted $\operatorname{Alg} \operatorname{Lat}(A)$. The algebra $A$ is called reflexive if $A=\operatorname{Alg} \operatorname{Lat}(A)$. An operator (or set of operators) $T$ is called reflexive if the weakly closed unital algebra it generates, $W(T)$, is reflexive.

D. Sarason proved that normal operators are reflexive, and that so are analytic Toeplitz operators [Sa1]. R. Olin and J. Thomson extended this result in 1979 to prove that all subnormal operators (i.e. restrictions of normal operators to invariant subspaces) are reflexive [OT]. Whilst their original proof has been somewhat simplified since then [Th1], [Th2], [Co1], to date all proofs have relied on an elaborate construction of "full analytic subspaces". We show that Thomson's work on bounded point evaluations [Th3] allows a much simpler proof (Theorem 1).

An $n$-tuple of operators $N=\left(N_{1}, \ldots, N_{n}\right)$ is called normal if each $N_{i}$ is normal, and $N_{i} N_{j}=N_{j} N_{i}$ for all $i, j$. The $n$-tuple $S=\left(S_{1}, \ldots, S_{n}\right)$ of operators on $\mathscr{H}$ is called subnormal if there is a Hilbert space $\mathscr{K}$ containing $\mathscr{H}$, and a normal $n$-tuple $\left(N_{1}, \ldots, N_{n}\right)$ on $\mathscr{K}$, such that each $N_{i}$ leaves $\mathscr{H}$ invariant, and $\left.N_{i}\right|_{\mathscr{H}}=S_{i}$. Just as in the cyclic case, a subnormal $n$-tuple is reflexive if its restriction to every cyclic subspace is. Moreover, any cyclic subnormal $n$-tuple is unitarily equivalent to $\left(M_{z_{1}}, \ldots, M_{z_{n}}\right)$ on $P^{2}(\mu)$ for some compactly supported measure $\mu$ on $\mathbb{C}^{n}$ (see $\S 2$ ). So studying reflexivity reduces to studying the spaces $P^{2}(\mu)$ and the $n$-tuple $S_{\mu}$ of multiplication by the variables.

A point $\lambda$ in $\mathbb{C}$ is called a bounded point evaluation for $P^{2}(\mu)$ if the functional of evaluating at $\lambda$, defined on the polynomials, is bounded 
on the space $P^{2}(\mu)$. If $\lambda$ is a bounded point evaluation, then there is a function $k_{\lambda}$ such that $p(\lambda)=\int p \overline{k_{\lambda}} d \mu$. Integration against this kernel function gives a well-defined meaning for $f(\lambda)$ for any function $f$ in $P^{2}(\mu)$, and we shall use this without further comment (this is a direct generalization of the usual practice of thinking of functions in the Hardy space $H^{2}$ both as functions on the unit circle with vanishing negative Fourier coefficients, and as analytic functions on the unit disk). The point $\lambda$ in $\mathbb{C}$ is called an analytic bounded point evaluation for $P^{2}(\mu)$ if $\lambda$ is in the interior of the bounded point evaluations, and for each $f$ in $P^{2}(\mu)$, the function sending $\zeta$ to $\int f \overline{k_{\zeta}} d \mu$ is analytic in a neighbourhood of $\lambda$.

The most important result about single subnormal operators since $S$. Brown's proof of the existence of invariant subspaces [Br] was Thomson's proof of the existence of analytic bounded point evaluations [Th3]. He showed that if $S_{\mu}$ is pure, all the bounded point evaluations are analytic bounded point evaluations, and that the kernel functions $k_{\lambda}$ span $P^{2}(\mu)$. We shall use this as one of the pillars of our proof of reflexivity of single subnormal operators.

The second pillar is the structure of $A(S)$, the weak-star (i.e. $\sigma$-weakly) closed algebra generated by the subnormal operator $S$. Conway and Olin [CO] showed that this algebra is isometrically isomorphic and weak-star homeomorphic to $P^{\infty}(\mu)$, the weak-star closure of the polynomials in $L^{\infty}(\mu)$, where $\mu$ is the scalar spectral measure for the minimal normal extension of $S$. Sarason has shown [Sa2] that $P^{\infty}(\mu)$ consists of an $L^{\infty}$ summand plus the space of bounded analytic functions on the interior of a certain set $\Sigma(\mu)$, called the Sarason hull.

The third pillar is that the algebra $A(S)$ is elementary, (also called $\left.\mathbb{A}_{1}\right)$ i.e. if $L$ is a weak-star continuous linear functional on $A(S)$, then there are vectors $x$ and $y$ such that $L(p(S))=\langle(S) x, y\rangle$ for any polynomial $p$. This idea first surfaced in $[\mathrm{Br}]$, and was proved in general in [OT]. The proof was refined in [Th2], and the state-of-theart proof is in $[\mathrm{BC}]$. One of the consequences is that $A(S)=W(S)$. See [Co1] for an exposition of all these results.

These three results (the existence of analytic bounded point evaluations, the representation of $A(S)$ as $P^{\infty}(\mu)$, together with Sarason's description of $P^{\infty}(\mu)$, and the factoring of weak-star continuous linear functionals) are fundamental to the modern theory of single subnormal operators. However, for subnormal tuples the first two results are in general false, and the validity of the third (whether the $\sigma$-weakly 
closed algebra a subnormal tuple generates is elementary) is open. We can make some progress if we impose (fairly stringent) restrictions, for example:

THeOREM 4. Let $\mu$ be a measure on $\mathbb{C}^{n}$, and let $\Omega$ be the set of analytic bounded point evaluations for $P^{2}(\mu)$. Suppose $\Omega$ has polynomially convex closure, and is either (i) strongly pseudoconvex or (ii) star-shaped. Suppose also that the span of the kernel functions corresponding to points of $\Omega$ is dense in $P^{2}(\mu)$. Then $S_{\mu}$ is reflexive.

In $\S 3$ we prove that if two isometries doubly commute then they are reflexive. An incomplete proof of this is given in [Pt1]. In $\S 4$ we give an example to show that non-commuting isometries need not be reflexive.

1. Single subnormal operators. The key part of the proof of reflexivity for subnormal operators is proving it for pure cyclic subnormal operators. It is well-known that any cyclic subnormal operator is unitarily equivalent to multiplication by the independent variable on some space $P^{2}(\mu)$, where $\mu$ is a compactly supported measure on $\mathbb{C}$. We shall denote this operator $S_{\mu}$; it is pure (i.e. has no normal summand) if $P^{2}(\mu)$ does not have $L^{2}\left(\left.\mu\right|_{E}\right)$ as a summand for any set $E$ of positive measure. For this, and other basic facts about subnormal operators, see J. Conway's book [Co1].

THEOREM 1. Pure cyclic subnormal operators are reflexive.

Proof. Assume the operator is $S_{\mu}$ for some $\mu$. Let $T$ be in $\operatorname{Alg} \operatorname{Lat}\left(A\left(S_{\mu}\right)\right)$.

(i) The kernel functions $k_{\lambda}$ are eigenvectors of $S_{\mu}^{*}$, so are left invariant by $T^{*}$. Therefore $T^{*}$ commutes with $S_{\mu}^{*}$ on each of the one-dimensional spaces spanned by some $k_{\lambda}$, and as their span is dense, $T^{*}$ commutes with $S_{\mu}^{*}$. Therefore $T$ commutes with $S_{\mu}$, and is given by multiplication by some function $\phi$ in $P^{2}(\mu) \cap L^{\infty}(\mu)$.

(ii) Because $A\left(S_{\mu}\right)$ is isometrically isomorphic and weak-star homeomorphic to $P^{\infty}(\mu)$, we must show $\phi$ is in $P^{\infty}(\mu)$; this requires showing that $\phi$ is analytic on the interior of $\Sigma(\mu)$.

Let $f$ be in $P^{2}(\mu)$. The closure of $\{p f: p$ a polynomial $\}$ is an invariant subspace for $S_{\mu}$, so must be $\phi$ invariant. Therefore one can approximate $\phi f$ by $p_{n} f$, where $p_{n}$ are polynomials; but this means one can approximate $\phi$ by $p_{n}$ in $L^{2}\left(|f|^{2} \mu\right)$, so $\phi$ is in $P^{2}\left(|f|^{2} \mu\right)$. 
Therefore $\phi$ is analytic on the set of analytic bounded point evaluations of $P^{2}\left(|f|^{2} \mu\right)$, for every $f$.

(iii) Let $\lambda$ be an arbitrary point in the interior of $\Sigma(\mu)$. Evaluation at $\lambda$ is weak-star continuous, so there are functions $x$ and $y$ in $P^{2}(\mu)$ such that $p(\lambda)=\int p x \bar{y} d \mu$. Therefore $\lambda$ is a bounded point evaluation for $P^{2}\left(|x|^{2} \mu\right)$, hence an analytic bounded point evaluation (as $S_{|x|^{2} \mu}$ is pure if $S_{\mu}$ is); therefore $\phi$ is analytic at $\lambda$, as desired.

To prove that all subnormal operators are reflexive is now fairly quick. For completeness, we include a proof based on that in Conway [Co1] Theorem VII.8.5; notice that the proof of the previous theorem allows one to skip completely sections VII.6 and VII.7 in [Co1].

THEOREM 2. All subnormal operators are reflexive.

Proof (Conway). (i) First, we show that reflexivity of cyclic subnormals implies it for all subnormals.

Let $S$ be subnormal on $\mathscr{H}$, with minimal normal extension $N$, and let $T$ be in $\operatorname{Alg} \operatorname{Lat}(A(S))$. Let $x$ and $y$ be vectors in $\mathscr{H}$ such that $x, y$ and $x+y$ are all separating vectors for the von Neumann algebra generated by $N$. Such vectors are dense in $\mathscr{H}$.

By hypothesis (and the separating requirement), on the cyclic spaces generated by $x, y$ and $x+y, T$ agrees with operators in $A(S)$, which we shall call respectively $X, Y$ and $Z$. So

$$
T(x+y)=Z(x+y)=T x+T y=X x+Y y .
$$

Therefore

$$
(X-Z) x=(Z-Y) y .
$$

Since this last vector is in both the space generated by $x$ and the space generated by $y$, both $X$ and $Y$ must agree on it. Therefore

$$
0=(X-Y)(X-Z) x=(X-Y)(Z-Y) y .
$$

Because both $x$ and $y$ are separating,

$$
0=(X-Y)(X-Z)=(X-Y)(Z-Y) \text {. }
$$

Adding then yields that $(X-Y)^{2}=0$, so $X=Y$.

(ii) For a normal operator $N$, the spectral theorem implies that any operator leaving $N$ 's reducing subspaces invariant must commute 
with it. So parts (ii) and (iii) of the proof of Theorem 1 prove that cyclic normal operators are reflexive, and hence all normal operators are.

(iii) To get rid of purity, note that if $S=N_{0} \oplus S_{1}$ is the decomposition of $S$ into a normal and pure part, and $T$ is in $\operatorname{Alg} \operatorname{Lat}(A(S))$, then $T=T_{0} \oplus T_{1}$ in $A\left(N_{0}\right) \oplus A\left(S_{1}\right)$. Because this last algebra is elementary, any weak-star continuous functional is of the form $\left(x_{0} \oplus x_{1}\right) \otimes\left(y_{0} \oplus y_{1}\right)$. If this annihilates $A(S),\left(y_{0} \oplus y_{1}\right)$ is orthogonal to the subspace generated by $A(S)\left(x_{0} \oplus x_{1}\right)$, and hence to $T\left(x_{0} \oplus x_{1}\right)$. Therefore $T$ is in $A(S)$, as desired.

2. Subnormal tuples. Many of the properties of subnormal operators carry over to subnormal tuples, but as we know of no source where they are written down, we list some here. The proofs are fairly straightforward generalizations of the proofs for single subnormal operators, so we shall not include them here. For other properties of subnormal tuples, see [CS] and [Co2]. We assume in what follows that $S=\left(S_{1}, \ldots, S_{n}\right)$ is a subnormal $n$-tuple on $\mathscr{H}$, with minimal normal extension $N$ on $\mathscr{K}$ (minimal means that $\mathscr{K}$ is the closed linear span of $\left.\left\{N_{1}^{* i_{1}} \ldots N_{n}^{* i_{n}} \xi: \xi \in \mathscr{H}, i_{1}, \ldots, i_{n} \in \mathbb{N}\right\}\right)$.

There is a measure $\mu$ supported on a compact subset $K$ of $\mathbb{C}^{n}$, and an isometric isomorphism and weak-star homeomorphism $\rho$ from $L^{\infty}(\mu)$ onto $W^{*}(N)$, the von Neumann algebra generated by $N$, taking $N_{i}$ onto the $i$ th coordinate function $z_{i}$. Spatially, $N$ is unitarily equivalent to the $n$-tuple of multiplication operators $M_{z}:=$ $\left(M_{z_{1}}, \ldots, M_{z_{n}}\right)$ on $L^{2}(\mu ; D)$ of square-summable functions from $K$ to $D$, a Hilbert space of possibly varying dimension. If $S$ is cyclic, it is unitarily equivalent to $S_{\mu}, M_{z}$ on $P^{2}(\mu)$ (this was first observed in [Ha]). Separating vectors for $W^{*}(N)$ are dense in both $\mathscr{K}$ and $\mathscr{H}$; and if $x$ and $y$ are separating vectors, and $\epsilon>0$, there is some $\alpha, 0<\alpha<\epsilon$, such that $x+\alpha y$ is also separating.

If $\phi$ in $L^{\infty}(\mu)$ satisfies $\rho(\phi(N)) \mathscr{H} \subseteq \mathscr{H}$, we will write $\phi(S)$ for $\left.\rho(\phi(N))\right|_{\mathscr{H}}$; it is then true that $\|\phi(S)\|=\|\phi(N)\|=\|\phi\|_{\infty}$. From this it follows that both $A(S)$ and $A(N)$ are naturally isometrically isomorphic and weak-star homeomorphic to $P^{\infty}(\mu)$. If $S$ is cyclic and $T$ is an operator that commutes with each $S_{i}$, then $T$ is multiplication by some bounded function in $P^{2}(\mu)$.

All normal $n$-tuples are reflexive. A subnormal $n$-tuple is reflexive if its restriction to every cyclic subspace is reflexive.

It is not known in general whether subnormal tuples are reflexive 
(even when a pair of commuting isometries), or whether weak-star continuous linear functionals on $A(S)$ can be factored as $x \otimes y$. The methods of the previous section, relying as they do on analyticity, can certainly not be applied to general subnormal tuples. To see how bad these can be, consider the following example.

ExAmple. Let $X$ be a compact subset of $\mathbb{C}$. The Banach algebra $R(X)$, the closure in $C(X)$ of the rational functions with poles off $X$, is doubly generated, as is well-known; choose one generator to be the coordinate function $z$, call the other generator $f$. Let l: $z \mapsto(z, f(z))$ map $X$ onto $K$, a subset of $\mathbb{C}^{2}$. Then $l^{*}$ gives an isomorphism from $P(K)$ to $R(X)$, and $l^{* *}$ gives an isomorphism from the measures on $X$ to the measures on $K$. Moreover, $K$ is polynomially convex, because the maximal ideal space of $P(K)$ is $l(X)=K$. Thus, $P^{2}(\mu)$, for $\mu$ a measure on $K$, is just as bad as $R^{2}(X, \nu)$ can be. In particular, there exists a measure $\mu$ on a polynomially convex set $K$ such that $P^{2}(\mu)$ has no $L^{2}$ summands, but

(i) the weak-star continuous point evaluations of $P^{\infty}(\mu)$ have no interior;

(ii) $P^{2}(\mu)$ has no bounded point evaluations at all [Bre].

However, the natural $P^{2}(\mu)$ spaces to study are those that do possess analytic structure. In particular, the obvious measures are volume and surface-area, and these clearly have analytic bounded point evaluations (via the Bergman and Szegö kernels). The difficulty lies in identifying $P^{\infty}(\mu)$. Notice that the proof of Theorem 1 yields the following for cyclic subnormal tuples:

THEOREM 3. Let $\mu$ be a measure on $\mathbb{C}^{n}$, and let $\Omega$ be the set of analytic bounded point evaluations for $P^{2}(\mu)$. Suppose

(a) the span of the kernel functions corresponding to points of $\Omega$ is dense in $P^{2}(\mu)$;

(b) $P^{2}(\mu) \cap H^{\infty}(\Omega)=P^{\infty}(\mu)$.

Then the subnormal $n$-tuple $S_{\mu}$ is reflexive.

As we said, the difficulty lies in checking (b). Here are two cases where it holds.

THEOREM 4. Suppose $\Omega$ has polynomially convex closure, and is either (i) strongly pseudoconvex or (ii) star-shaped. Then, in the previous theorem, condition (a) implies condition (b). 
Proof. (i) Let $f$ be in $P^{2}(\mu) \cap H^{\infty}(\Omega)$. Because $\Omega$ is strongly pseudoconvex, there are functions $f_{j}$, analytic on a neighborhood of $\bar{\Omega}$, converging to $f$ uniformly on compacta, and satisfying $\left\|f_{j}\right\|_{\infty} \leq$ $\|f\|_{\infty}[\mathbf{C R}]$ - see also [BF]. As $\bar{\Omega}$ is polynomially convex, the $f_{j}$ 's in turn can be uniformly approximated by polynomials $p_{j, k}$. As the ball of $P^{\infty}(\mu)$ is weak-star compact, some subsequence of $p_{j, j}$ will converge weak-star to some function $g$ in $P^{\infty}(\mu)$. But

$$
\int g \overline{k_{\lambda}} d \mu=g(\lambda)=\lim p_{j j}(\lambda)=\int f \overline{k_{\lambda}} d \mu
$$

for all $\lambda$ in $\Omega$, so $f$ is in $P^{\infty}(\mu)$.

The reverse inclusion is obvious.

(ii) Assume for simplicity that $\Omega$ is star-shaped with respect to zero. Then the above argument works, with the obvious choice for $f_{j}$ of $f\left(r_{j} z\right)$, where $r_{j}$ increases to 1 .

Case (ii) above generalizes the results of M. Ptak in [Pt2].

3. Doubly commuting isometries. An $n$-tuple of operators $\left(T_{1}, \ldots, T_{n}\right)$ is said to doubly commute if, for any distinct indices $i$ and $j, T_{i} T_{j}=T_{j} T_{i}$ and $T_{i}^{*} T_{j}=T_{j} T_{i}^{*}$. In this section we prove that any pair of doubly commuting isometries is reflexive. That single isometries are reflexive was first proved by J. Deddens [De]. In [Pt1], Ptak claims to prove that a pair of doubly commuting isometries is reflexive; however, his proof contains a gap.

Specifically, let $S$ be the shift on $H^{2}(\mathscr{H})$, the space of squaresummable analytic functions with values in the Hilbert space $\mathscr{H}$ (for information on shifts of multiplicity greater than one, which can be represented as multiplication by $z$ on some $H^{2}(\mathscr{H})$, see the book [Sz-NF]). Let $T_{0}$ be an operator on $\mathscr{H}$, and let $T$ be the operator in $\{S\}^{\prime}=H^{\infty}(B(\mathscr{C}))$ defined by $T(z)=T_{0}$ for all $z$ in the circle. Then in [Pt1], Proposition 4, Ptak asserts that if $T_{0}$ has property $C_{\infty}$, then $\{S, T\}$ has property $C$ (see definitions below), and says the proof is a small modification of the proof of [Wo], Lemma 2. But Wogen's proof, which he gives for the case that $T_{0}$ is positive, can only be generalized to reductive operators $T_{0}$ (see Lemma 5 below). We note that if $T_{0}$ is the unilateral shift, Ptak's assertion is equivalent to the following question in function theory:

Let $f_{1}, f_{2}$ be two functions in $H^{2}\left(\mathbb{T}^{2}\right)$. Does there necessarily exist $f_{0}$ in $H^{2}\left(\mathbb{T}^{2}\right)$ such that $\left|f_{0}\right|^{2}=\left|f_{1}\right|^{2}+\left|f_{2}\right|^{2}$ almost everywhere on the torus? 
Definitions. If $T$ is an operator on the Hilbert space $\mathscr{H}$, then $T^{(n)}$ acting on $\mathscr{H}^{(n)}$ is the direct sum of $n$ copies of $T$ with itself. If $\mathscr{T}$ is a subset of $B(\mathscr{H})$, and $x$ is a vector in $\mathscr{H}$, then $C(\mathscr{T}, x)$ is the smallest closed subspace containing $x$ and invariant under every element of $\mathscr{T}$. A subset $\mathscr{T}$ of $B(\mathscr{H})$ has property $C$ (introduced in [Wo]) if, for every positive integer $n$ and vector $\eta$ in $\mathscr{H}^{(n)}$, there is a vector $\xi$ in $\mathscr{H}$ and a unitary operator $U: C\left(\mathscr{T}^{(n)}, \eta\right) \rightarrow C(\mathscr{T}, \xi)$ such that, for every $T$ in $\mathscr{T}$,

$$
\left.U T^{(n)}\right|_{C\left(\mathscr{T}^{(n)}, \eta\right)} U^{*}=\left.T\right|_{C(\mathscr{T}, \xi)} \cdot
$$

If this also holds for $n=\infty$, say that $\mathscr{T}$ has property $C_{\infty}$.

The proof of the following lemma is based on [Wo], Lemma 2, but we include it for completeness. A normal operator is reductive if every invariant subspace is also invariant for its adjoint. In terms of the scalar spectral measure $\mu$, this is equivalent to saying that $P^{\infty}(\mu)=L^{\infty}(\mu)$.

LEMMA 5. Let $S$ be the unilateral shift on $H^{2}(\mathscr{H})$, and let $\mathscr{T}_{0}$ be a finite set of commuting normal operators on $\mathscr{H}$ that are all reductive. Extend each operator $T_{0}$ in $\mathscr{T}_{0}$ to an operator $T$ on $H^{2}(\mathscr{H})$, by defining $T(z)=T_{0}$ for all $z$ in the circle; call the new collection $\mathscr{T}$. Then $A(S, \mathscr{T})$ has property $C_{\infty}$.

Proof. Let $\eta$ be in $H^{2}(\mathscr{H})^{(n)}$, for some $1 \leq n \leq \infty$. Let $\mathscr{M}=$ $C\left(\left\{S^{(n)}, \mathscr{T}^{(n)}\right\}, \eta\right)$ and $\mathscr{M}_{0}=\mathscr{M} \ominus S^{(n)} \mathscr{M}$. For each $T$ in $\mathscr{T}, S^{(n)} \mathscr{M}$ is invariant under $T^{(n)}$, so $H^{2}(\mathscr{H})^{(n)} \ominus S^{(n)} \mathscr{M}$ is invariant for $T^{(n)^{*}}$. Because $T^{(n)}$ is reductive, $H^{2}(\mathscr{H})^{(n)} \ominus S^{(n)} \mathscr{M}$ is also invariant for $T^{(n)}$, so $T^{(n)} \mathscr{M}_{0}$ is contained in $\mathscr{M}_{0}$. Therefore $\mathscr{M}_{0}$ is a reducing subspace for each $T^{(n)}$, and $\left.T^{(n)}\right|_{\mathscr{M}_{0}}$ is always normal.

The space $\mathscr{M}_{0}$ is precisely $C\left(\mathscr{T}^{(n)}, P_{\mathscr{M}_{0}} \eta\right)$, where $P_{\mathscr{\mathscr { O }}_{0}}$ is the orthogonal projection onto $\mathscr{M}_{0}$. By the spectral theorem for normal tuples, and as each $T$ is just a direct sum of countably many copies of $T_{0}$, there is a $\mathscr{T}_{0}$-cyclic subspace $\mathscr{N}_{0}$ of $\mathscr{H}$ and a unitary map $U_{0}$ from $\mathscr{M}_{0}$ onto $\mathscr{N}_{0}$ such that $\left.U_{0} T^{(n)}\right|_{\mathscr{M}_{0}} U_{0}^{*}=\left.T_{0}\right|_{\mathcal{N}_{0}}$ for each $T$ in $\mathscr{T}$. Now extend this to a map from $\mathscr{M}$ into $H^{2}(\mathscr{H})$ by embedding $\mathscr{N}_{0}$ into the constant functions of $H^{2}(\mathscr{H})$ (i.e. the kernel of $S^{*}$ ), and defining $U\left(S^{(n)^{k}} m_{0}\right)=z^{k} U_{0}\left(m_{0}\right)$ for $m_{0}$ in $\mathscr{M}_{0}$. As the range of $U$ is $A(S, \mathscr{T})$-cyclic, $A(S, \mathscr{T})$ has property $C_{\infty}$, as required.

We also need the following lemma. It is also proved in [Pt1]. 
LemMa 6. Let $S$ be the unilateral shift on $H^{2}(\mathscr{H})$, and let $\mathscr{T}_{0}$ be a finite commuting set of operators on $\mathscr{H}$ such that $\operatorname{Alg} \operatorname{Lat}\left(\mathscr{T}_{0}\right)=A\left(\mathscr{T}_{0}\right)$. Extend each operator $T_{0}$ in $\mathscr{T}_{0}$ to an operator $T$ on $H^{2}(\mathscr{H})$, by defining $T(z)=T_{0}$ for all $z$ in $\mathscr{T}$; call the new collection $\mathscr{T}$. Then $\operatorname{Alg} \operatorname{Lat}(S, \mathscr{T})=W(S, \mathscr{T})=A(S, \mathscr{T})$.

Proof. Let $R$ be in $\operatorname{Alg} \operatorname{Lat}(S, \mathscr{T})$. If $k_{\lambda}(z)$ is the kernel function $1 /(1-\bar{\lambda} z)$, then $\left\{k_{\lambda} . \mathscr{H}\right\}$ is the kernel of $(S-\lambda)^{*}$, and is in $\operatorname{Lat}\left(S^{*}, \mathscr{T}^{*}\right)$. Therefore $R^{*}$ leaves it invariant, and so commutes with $S^{*}$ on it. As these spaces span $H^{2}(\mathscr{H}), R$ commutes with $S$. Therefore $R$ can be written as $R=\sum_{k=0}^{\infty} z^{k} R_{k}$, where each $R_{k}$ maps $\mathscr{H}$ to $\mathscr{H}$, and the series converges almost uniformly on the disk, and radially it converges strongly almost everywhere (see [Sz-NF]).

Let $\mathscr{M}$ be an arbitrary $\mathscr{T}_{0}$-invariant subspace. Then $H^{2}(\mathscr{M})$ is $A(S, \mathscr{T})$-invariant, so $R$-invariant. Therefore for almost every $w$ in $\mathbb{T}, R(w)=\lim _{r \uparrow 1} \sum_{k=0}^{\infty}(r w)^{k} R_{k}$ is in $\operatorname{Alg} \operatorname{Lat}\left(\mathscr{T}_{0}\right)$. So if $\xi$ is in $\mathscr{M}$ and $\eta$ is in $\mathscr{M}^{\perp}$,

$$
\left(\sum_{k=0}^{\infty} w^{k} R_{k} \xi, \eta\right)=0 \text { a.e. }
$$

The left-hand side is a bounded analytic function of $w$, so it vanishes for all $w$ in the unit disk. In particular, for $w=0$, this gives that $R_{0}$ is in $\operatorname{Alg} \operatorname{Lat}\left(\mathscr{O}_{0}\right)$. Hence $\sum_{k=0}^{\infty} w^{k} R_{k+1}$ is in $\operatorname{Alg} \operatorname{Lat}\left(\mathscr{T}_{0}\right)$ for almost every $w$, and by induction each $R_{k}$ is.

Let

$$
R_{r}=\sum_{k=0}^{\infty} r^{k} z^{k} R_{k}
$$

For $0<r<1$, the partial sums $\sum_{k=0}^{N} r^{k} z^{k} R_{k}$ are all in $A(S, \mathscr{T})$, and converge to $R_{r}$ in norm. Moreover, as $\left\{R_{r}: 0<r<1\right\}$ is uniformly bounded, $R_{r}$ converges to $R$ in both the weak and $\sigma$-weak operator topologies.

THEOREM 7. Let $T=\left(T_{1}, T_{2}\right)$ be a pair of doubly commuting isometries on the Hilbert space $\mathscr{H}$. Then $\operatorname{Alg} \operatorname{Lat}(T)=W(T)=A(T)$.

Proof. For each isometry $T_{i}, \mathscr{H}$ has a Wold decomposition, i.e. it decomposes into two reducing subspaces, on the first of which the operator is a pure shift, and on the second of which it is unitary. As the first space is the span of the kernels of $\left(T_{i}^{*}\right)^{n}$, it is left invariant by everything that commutes with $T_{i}^{*}$; so the double commutativity 
hypothesis yields that each of these spaces is also reducing for the other isometry. Therefore $\mathscr{H}$ decomposes into 4 spaces, on which each $T_{i}$ is either unitary or a pure shift.

Moreover, the spaces where we have a shift $S$ and a unitary $U$ decompose further. By multiplying by the spectral projection for $U$ corresponding to, say, the right semi-circle, $U$ decomposes into the direct sum of two reductive operators, and because $S$ commutes with $U$, it also commutes with this decomposition.

Therefore $\mathscr{H}$ decomposes into (up to) 6 reducing subspaces, on which $T_{1}, T_{2}$ are either

(i) both unitary, or

(ii) one a pure shift, the other a reductive unitary, or

(iii) both pure shifts.

We claim that in each case the pair is reflexive, the weakly and $\sigma$ weakly closed algebras they generate coincide, and that this algebra is elementary. This implies that on the direct sum of the subspaces the pair is reflexive and the two algebras coincide.

(i) We have already remarked that normal tuples are reflexive. That the algebra $A(T)$ is elementary follows from the spectral theorem.

(ii) This follows from Lemmata 5 and 6 , because property $C_{\infty}$ implies $A(T)$ is elementary.

(iii) Because $T_{1}$ and $T_{2}$ doubly commute, the space decomposes into a direct sum of copies of $H^{2}\left(\mathbb{T}^{2}\right)$, where the operators are multiplication by the coordinate functions. The pair is reflexive (and $A(T)=W(T)$ ) by Lemma 6. That $A(T)$ is elementary follows from the theorem of H. Bercovici and D. Westwood that any function $h$ in $L^{1}\left(\mathbb{T}^{n}, \sigma\right)$, where $\sigma$ is Lebesgue measure, can be factored as $h=f g$, for some $f$ in $H^{2}\left(\mathbb{T}^{n}\right)$ and $g$ in $L^{2}\left(\mathbb{T}^{n}, \sigma\right)$ [BW].

4. Non-commuting isometries. We finish with an example to show that non-commuting isometries need not be reflexive.

EXAMPLE 8. Let $S$ be the unilateral shift, and $U$ the diagonal operator with first two entries -1 , other entries 1 .

$$
S=\left(\begin{array}{cccc}
0 & 0 & 0 & \ldots \\
1 & 0 & 0 & \ldots \\
0 & 1 & 0 & \ldots \\
\vdots & \vdots & \vdots & \ddots
\end{array}\right), \quad U=\left(\begin{array}{cccc}
-1 & & & \\
& -1 & & \\
& & 1 & \\
& & & \ddots .
\end{array}\right)
$$


Let $A$ be the weakly closed algebra generated by $S$ and $U$. We claim that $A$ is not reflexive.

By Beurling's theorem, the invariant subspaces of $S$, thought of as an operator on the Hardy space $H^{2}$, are the spaces $u H^{2}$, where $u$ is an inner function. So $\operatorname{Lat}(A)$ consists of those $u H^{2}$ which are $U$-invariant. Let $u(z)=\sum_{n=0}^{\infty} a_{n} z^{n}$. If $a_{0}=a_{1}=0$, then $u H^{2}$ is clearly $U$-invariant.

Suppose $a_{0} \neq 0$, and $u H^{2}$ is in $\operatorname{Lat}(A)$. Then applying the operator $(I-U)-\frac{a_{1}}{a_{0}}(I-U) S$ to $u$, one gets that the constant function $2 a_{0}$ is in $u H^{2}$, so $u H^{2}$ must be all of $H^{2}$. Similarly, if $a_{0}=0$ and $a_{1} \neq 0$, then the operator $\frac{1}{2 a_{1}}(I-U)$ applied to $u$ gives the function $z$, so $u H^{2}=z H^{2}$. Therefore $\operatorname{Lat}(A)=\left\{z^{2} u H^{2}: u\right.$ inner $\} \cup\left\{z H^{2}\right\} \cup$ $\left\{H^{2}\right\} \cup\{0\}$.

Let $R$ be the rank-one diagonal operator

$$
R=\left(\begin{array}{lll}
1 & & \\
& 0 & \\
& & \ddots
\end{array}\right) \text {. }
$$

Clearly $R$ is in $\operatorname{Alg} \operatorname{Lat}(A)$. We shall show it is not in $A$.

Let $p(U, S)$ be a non-commuting polynomial in $U$ and $S$. Because of the identities $U^{2}=I, U S^{2}=S^{2}$ and $U S U S=S U S$, $p(U, S)$ must have the reduced form

$$
p(U, S)=q_{1}(S)+q_{2}(S) U+q_{3}(S) U S+q_{4}(S) U S U .
$$

So if $\left\{e_{n}\right\}_{n=0}^{\infty}$ is the usual orthonormal basis for $H^{2},\left\langle p(U, S) e_{0}, e_{0}\right\rangle$ $=\left\langle p(U, S) e_{1}, e_{1}\right\rangle$. As $A$ is the weak closure of polynomials in $U$ and $S$, anything in $A$ must have its first two diagonal entries equal. Therefore $R$ is not in $A$, as claimed.

Note that on a finite-dimensional space, all subnormal operators are normal and reductive. Therefore the weakly-closed algebra generated by any set of them is a von Neumann algebra, and hence reflexive.

\section{REFERENCES}

[BF] E. Bedford and J. E. Fornaess, Approximation on pseudoconvex domains, in Complex Approximation, ed. B. Aupetit, Birkhäuser, 1980.

[BC] H. Bercovici and J. B. Conway, A note on the algebra generated by a subnormal operator, in Operator Theory, Adv. and Appl., 32 (1988), 53-56.

[BW] H. Bercovici and D. Westwood, The factorization of functions in the polydisc, to appear. 
[Bre] J. E. Brennan, Invariant subspaces and rational approximation, J. Funct. Anal., 7 (1971), 285-310.

[Br] S.W. Brown, Some invariant subspaces for subnormal operators, Integral Equations Operator Theory, 1 (1978), 310-333.

[CR] B. Cole and M. Range, A-measures on complex manifolds and some applications, J. Funct. Anal., 11 (1972), 393-400.

[Co1] J. B. Conway, The theory of subnormal operators, Amer. Math. Soc., Providence, RI, 1991.

[Co2] _ Towards a functional calculus for subnormal tuples: the minimal normal extension, in the Proceedings of the AMS Summer Institute in Operator Theory, held in New Hampshire, 1988.

[CO] J. B. Conway and R. F. Olin, A functional calculus for subnormal operators II, Mem. Amer. Math. Soc., vol. 184, 1977.

[De] J. A. Deddens, Every isometry is reflexive, Proc. Amer. Math. Soc., 28 (1971), 509-512.

[Ha] W. W. Hastings, Commuting subnormal operators simultaneously quasisimilar to unilateral shifts, Illinois J. Math., 22 (1978), 506-519.

[OT] R. F. Olin and J. E. Thomson, Algebras of subnormal operators, J. Funct. Anal., 37 (1980), 271-301.

[Pt1] M. Ptak, Reflexivity of pairs of isometries, Studia Math., 83 (1986), 47-55.

[Pt2] - Reflexivity of multiplication operators in certain domains in $C^{n}$, Bull. Polish Acad. Sci. Math., 37 (1989), 217-220.

[Sa1] D. Sarason, Invariant subspaces and unstarred operator algebras, Pacific J. Math., 17 (1966), 511-517.

[Sa2] _ Weak-star density of polynomials, J. Reine Angew. Math., 252 (1972), $1-15$.

[Sz-NF] B. Sz-Nagy and C. Foias, Harmonic Analysis of Operators on Hilbert Space, North-Holland, Amsterdam, 1970.

[Th1] J. E. Thomson, Invariant subspaces for subnormal operators, in Surveys of some Recent Results in Operator Theory, ed. J. B. Conway and B. B. Morrel, Pitman, Harlow, 1988.

[Th2] _ Factorization over algebras of subnormal operators, Indiana Math. J., 37 (1988), 191-199.

[Th3] _ Approximation in the mean by polynomials, Ann. of Math., 133 (1991), 477-507.

[Wo] W. R. Wogen, Quasinormal operators are reflexive, Bull. London Math. Soc., 31 (1979), 19-22.

Received January 15, 1992. The author was partially supported by National Science Foundation grant DMS 9102965.

WASHINGTON UNIVERSITY

ST. LouIs, MO 63130

E-mail address: mccarthy@jezebel.wustl.edu 


\title{
PACIFIC JOURNAL OF MATHEMATICS
}

Founded by

\author{
E. F. BECKenBaCH (1906-1982) F. WolF (1904-1989)
}

\section{EDITORS}

Sun-Yung A. Chang

(Managing Editor)

University of California

Los Angeles, CA 90024-1555

chang@math.ucla.edu

F. Michael Christ

University of California

Los Angeles, CA 90024-1555

christ@math.ucla.edu

Herbert Clemens

University of Utah

Salt Lake City, UT 84112

clemens@math.utah.edu

\author{
R. FInN
}

THOMAS ENRIGHT

University of California, San Diego

La Jolla, CA 92093

tenright@ucsd.edu

Nicholas ERColani

University of Arizona

Tucson, AZ 85721

ercolani@math.arizona.edu

Stanford University

Stanford, CA 94305

finn@gauss.stanford.edu

VAUghan F. R. JoNES

University of California

Berkeley, CA 94720

vfr@math.berkeley.edu

\section{SUPPORTING INSTITUTIONS}

STEVEN KeRCKHOFF

Stanford University

Stanford, CA 94305

spk@gauss.stanford.edu

MARTIN ScharLemanN

University of California

Santa Barbara, CA 93106

mgscharl@henri.ucsb.edu

HAROLd STARK

University of California, San Diego La Jolla, CA 92093

V. S. VARADARAJAN

University of California

Los Angeles, CA 90024-1555

vsv@math.ucla.edu

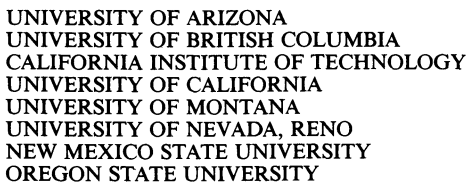

The Supporting Institutions listed above contribute to the cost of publication of this Journal, but they are not owners or publishers and have no responsibility for its content or policies.

Mathematical papers intended for publication in the Pacific Journal of Mathematics should be in typed form or offset-reproduced (not dittoed), double spaced with large margins. Please do not use built up fractions in the text of the manuscript. However, you may use them in the displayed equations. Underline Greek letters in red, German in green, and script in blue. The first paragraph must be capable of being used separately as a synopsis of the entire paper. In particular it should contain no bibliographic references. Please propose a heading for the odd numbered pages of less than 35 characters. Manuscripts, in triplicate, may be sent to any one of the editors. Please classify according to the 1991 Mathematics Subject Classification scheme which can be found in the December index volumes of Mathematical Reviews. Supply name and address of author to whom proofs should be sent. All other communications should be addressed to the managing editor, or Julie Honig, University of California, Los Angeles, California 90024-1555.

There are page-charges associated with articles appearing in the Pacific Journal of Mathematics. These charges are expected to be paid by the author's University, Government Agency or Company. If the author or authors do not have access to such Institutional support these charges are waived. Single authors will receive 75 free reprints; joint authors will receive a total of 100 free reprints. Additional copies may be obtained at cost in multiples of 50 .

The Pacific Journal of Mathematics (ISSN 0030-8730) is published monthly except for July and August. Regular subscription rate: $\$ 200.00$ a year (10 issues). Special rate: $\$ 100.00$ a year to individual members of supporting institutions.

Subscriptions, orders for numbers issued in the last three calendar years, and changes of address should be sent to Pacific Journal of Mathematics, P.O. Box 969, Carmel Valley, CA 93924, U.S.A. Old back numbers obtainable from Kraus Periodicals Co., Route 100, Millwood, NY 10546.

The Pacific Journal of Mathematics at P.O. Box 969, Carmel Valley, CA 93924 (ISSN 0030-8730) is published monthly except for July and August. Second-class postage paid at Carmel Valley, California 93924, and additional mailing offices. Postmaster: send address changes to Pacific Journal of Mathematics, P.O. Box 969, Carmel Valley, CA 93924.

PUBLISHED BY PACIFIC JOURNAL OF MATHEMATICS, A NON-PROFIT CORPORATION

This publication was typeset using $\mathcal{A} \mathcal{M S}-\mathrm{T}_{\mathrm{E}} \mathrm{X}$, the American Mathematical Society's $\mathrm{T}_{\mathrm{E}} \mathrm{X}$ macro system. Copyright (c) 1993 by Pacific Journal of Mathematics 


\section{PACIFIC JOURNAL OF MATHEMATICS}

Volume $161 \quad$ No. $2 \quad$ December 1993

On the method of constructing irreducible finite index subfactors of 201 Popa

FLORIN PETRE BOCA

Brownian motion and the heat semigroup on the path space of a

233 compact Lie group

JAY BARRY EPPERSON and TERRY M. LOHRENZ

Horizontal path spaces and Carnot-Carathéodory metrics

255

ZHONG GE

Biholomorphic convex mappings of ball in $\mathbb{C}^{n}$

SHENG GONG, SHI KUN WANG and QI HUANG YU

The Temperley-Lieb algebra at roots of unity

FREDERICK MiCHAEL GOODMAN and HANS WENZL

Jordan analogs of the Burnside and Jacobson density theorems

LUZIUS GRÜNENFELDER, M. OLMLADIČ and HEYDAR RADJAVI

Elliptic representations for $\mathrm{Sp}(2 n)$ and $\mathrm{SO}(n)$

REBECCA A. Herb

Reflexivity of subnormal operators

JOHN MCCARTHY

Knotting trivial knots and resulting knot types

KiMiHiko MOTEGI

Commutativity of selfadjoint operators

MitsURU UCHIYAMA

Correction to: "One-dimensional Nash groups" 